International Journal of Wireless \& Mobile Networks (IJWMN) Vol. 6, No. 1, February 2014

\title{
ZigBEE BASED VOICE CONTROLLED WIRELESS SMART HOME SYSTEM
}

\author{
Thoraya Obaid, Haliemah Rashed, Ali Abu El Nour, Muhammad Rehan, \\ Mussab Muhammad Saleh, and Mohammed Tarique \\ Department of Electrical Engineering, Ajman University of Science and Technology, \\ Fujairah, United Arab Emirates
}

\begin{abstract}
In this paper a voice controlled wireless smart home system has been presented for elderly and disabled people. The proposed system has two main components namely (a) voice recognition system, and (b) wireless system. LabView software has been used to implement the voice recognition system. On the other hand, ZigBee wireless modules have been used to implement the wireless system. The main goal of this system is to control home appliances by using voice commands. The proposed system can recognize the voice commands, convert them into the required data format, and send the data through the wireless transmitter. Based on the received data at the wireless receiver associated with the appliances desired switching operations are performed. The proposed system is a low cost and low power system because ZigBee is used. Additionally the proposed system needs to be trained of voice command only once. Then the system can recognize the voice commands independent of vocabulary size, noise, and speaker characteristics (i.e., accent).
\end{abstract}

\section{KEYWORDS}

Wireless, smart home, voice recognition, ZigBee, low power, low-cost, LabView,

\section{INTRODUCTION}

Home automation system has been around for more than a decade [1]. The main concept is to form a network connecting the electrical and electronic appliances in a house. This is a growing technology, which has changed the way people live. According to the data published by the market research and market intelligence firm ABI about 4 million home automation systems were sold globally in 2013 [8]. It is also estimated by the same organization that 90 million homes worldwide will employ home automation systems by the end of 2017. There have been several commercial and research versions of smart home system introduced and built $[2,3,4,5,6]$. But, none of the versions has broken through the mainstream yet other than security systems [7]. Smart home systems have captured many disparate technologies so far and products have been in the market for more than one decade. Many companies have entered in this field including Google. Google has announced an ambitious project named Android@Home [15] for smart home platforms. Despite over a decade of disparate activity in the industry no company has yet succeeded to launch home automation as a popular technology. The reasons of this failure have been comprehensively studied and listed in $[1,16,17]$. Some of the reasons are as follows: (a) cost: the existing systems are expensive and are owned by rich family with large house and estates, (b) difficult to install: expert professionals are needed to install and configure the system, (c) difficult to use: the control interfaces have poor quality and are not user friendly, (d) vendor dependency:

DOI : 10.5121/ijwmn.2014.6104 
International Journal of Wireless \& Mobile Networks (IJWMN) Vol. 6, No. 1, February 2014

need to use separate systems for different companies' appliances, (e) less functionality: most of the system can either monitor or control the functions, and (f) not customized: most of the systems are not customized with the needs of the users. In addition to the above mentioned applications there are also some other reasons including security issues and multi-user problems $[18,19]$.

Wireless communication based home automation system has gained a high momentum for the last couple of years. Wireless communication reduces the complexity related to the installation and maintenance compared to its wired counterpart. A typical wireless home automation system comprises battery operated and low power wireless sensors and actuators. Bluetooth, WiFi, and ZigBee are the popular choice for the backbone of such systems. Wireless network based smart home systems have become very popular as they provide comfort, security, and safety. Moreover, they support remote monitoring facilities. The availability of cheap wireless sensors, actuators, and modules has reduced the gap between the luxury and mass market segmentations of home automation technologies. However, wireless home automation system has some limitations too. The hostile radio channel, resource limitation, and mobility impose challenges for wireless home automation systems. Despite these limitations several organization and companies have developed wireless home automation system for diversified applications namely light control, remote control, smart energy, remote care, security and safety [21]. Especially, this industry has changed drastically since the introduction of cheap computers and laptops. The user interfaces of the home automation systems are much cheaper and user friendly now [20]. A typical wireless home automation system should have to deal with the following constraints: (a) high node density, (b) multipath radio wave propagation, (c) high interference, (d) multihop end-to-end connectivity, (e) dynamic topology, (f) various traffic patterns, (g) internet connectivity, and (h) secured communication. The major challenge is to deal with these constraints by using nodes that not only have limited memory and processing power, but also have limited operating life.

A typical wireless home automation system consists of two main parts namely (a) communication protocols, and (b) the user interfaces. The communication protocols are used for getting data to and from the home appliances. The user interfaces are used for monitoring and controlling them. There have been many solutions proposed for wireless home automation industry in the past few years. Some of them include (i) Z-wave, (ii) Insteon, (iv) Waveins, and (v) IP based solutions. The Z-wave is a wireless solution developed by Sigma Design. This wireless protocol has been promoted by the Z-wave alliance [12]. The main purpose of the Z-wave is to ensure a reliable transmission of short messages from a control unit to one or more nodes in the networks. Insteon is a home automation solution developed by SmartLabs and promoted by the Insteon Alliance. The major feature of Insteon is that it defines mesh topology composed of radio frequency (RF) link and power line link. The nodes can be RF only or power line links only or can support both types of communication. Waveins is a low power wireless protocol developed for controlling and monitoring appliances in a home. It is currently managed and promoted by Wavenis Open Standard Alliance [22]. This protocol defines physical, link, and network layers. Wavenis services can be accessed from the upper layer through an application programming interface. The IP-Based solutions have been initiated by Low Power Wireless Personal Area Network Group of Internet Engineering Task Force (IETF). This working group has defined mechanism for transmission of IPv6 packets on top of IEEE 802.15.4 networks. These networks have been named as LowPAN. The LowPAN follows the mesh topology and a routing protocol is used for its operation. The work on LowPAN is still in its infancy level and it is predicted that it will be an emerging technology for wireless home automation system in future.

Recently, ZigBee based solutions have drawn considerable attentions in the wireless home automation industry. This technology was developed by the ZigBee Alliance for low-data rate and short-range applications. ZigBee was designed for a suite of high level communication protocols used to create personal area networks. The initial version of ZigBee was based on IEEE 802.15.4 
International Journal of Wireless \& Mobile Networks (IJWMN) Vol. 6, No. 1, February 2014

standard. It operates in the $868 \mathrm{MHz}, 915 \mathrm{MHz}$, and $2.4 \mathrm{GHz}$ bands in Europe, North America, and worldwide respectively. The ZigBee protocol stacks composed of four layers namely physical layer, network layer, medium access control, and application layer. The physical layer and the medium access control layer are based on IEEE 802.15.4 standard. The ZigBee Alliance has defined the application layer and network layer. The ZigBee defines three roles for the devices namely (i) co-ordinator, (ii) router, and (iii) end device. The co-ordinator and router have more functionality compared to the end devices. The ZigBee end devices can transmit data over longer distances via the router devices. The network layer supports both addressing and routing for tree and mesh topologies. In tree topology the coordinator acts as root. In mesh topology routes are discovered and maintained on-demand. The Ad hoc On-Demand Distance Vector (AODV) [23] has been chosen as the routing protocol in ZigBee networks. Two routing strategies are used namely point-to-point, and many-to-one. There are several application profiles in ZigBee. One of the profiles is Home Automation Public Application Profile, which makes ZigBee a suitable technology for home automation systems [24]. This application profile defines device descriptions, commands, and attributes for ZigBee applications in residential and commercial environments. Some of the applications of ZigBee include residential and commercial lighting, HVAC, security, wireless light switches, electrical meters, traffic management systems, and other consumer and industrial equipment that require short-range wireless transfer of data at relatively low rate. Considering all the above mentioned advantages we have selected ZigBee.

Voice controlled home automation systems have drawn considerable attention in the recent years. Initially, home automation systems were designed for the people seeking luxury and sophisticated home. But, there was always a need to develop home automation system for the people with special needs like the elderly and the disabled. According to a report published by the World Health Organization (WHO) around 785 million people of 15 years and older live with disability [49]. Of these, the World Health Survey reports that 110 million people have significant difficulties in functioning. Another report published by the Population Division of United Nations show that about $10 \%$ of the World's population is older than 60 years and it is estimated that this figure will reach up to $21 \%$ by 2050 [50]. In order to assist the old people and the people with disability home automation technologies are adopting voice control or voice recognition techniques. The main idea is to control and monitor home appliances by using voice command. The motivation behind this work is also the same. The rest of the paper is organized as follows. Section 2 presents some related works. System model has been presented in Section 3. Section 4 contains system operation, circuit details, and implementations. Finally, this paper is concluded with Section 5.

\section{RELATED WORKS}

One of the early experimental works on the ZigBee based home automation system was presented in [25]. This system was capable of monitoring door and window, smoke, gas leak, and water flooding in a home from remote location. Some simple control systems such as operating a valve and sending signal to security network have also been associated with this application. A ZigBee based home network system to track a user has been proposed in [26]. This system periodically tracks a user by using three systems namely Indoor Positioning System (IPS-M), Indoor Positioning System Infrastructure (IPS-M), and Indoor Positioning System Gateway (IPS-G). In ZigBee based wireless home automation system a gateway is an important component. One of such gateway architecture has been proposed in [27] to interconnect Digital Living Network Alliance (DLNA) compliant home appliances and a ZigBee network. In a similar work [28], another type of gateway architecture has been proposed to connect a low-rate home work with the internet. A user can control the home appliances via internet from a remote location through this gateway. A ZigBee based power monitoring system (PMS) has been proposed in [29]. In addition 
to ZigBee wireless communication the PMS also utilizes Digital Signal Processing (DSP) and Web services. DSP is used for real-time power parameters computation and Web Services are used for the communication infrastructure among distributed systems across a network. The proposed system has been constructed and validated for the power management in a campus. Another power management system has been proposed in [30]. This system stores the measurement data of current and voltage of the electric outlets in an embedded board. It can detect any overload in the system and send signal to a circuit breaker to turn off the power. A real-time home security system has been proposed in [31]. The system is able to detect intruder in a home and send messages via GSM network. The system also can receive instruction from a remote location to control the house appliances. A wireless smart home system based on ZigBee has been introduced in [32]. The system composed of three main components (i) home server with GSM module; (ii) intelligent environment detection sensor modules, and (iii) intelligent home appliances. By using these modules a home can be monitored remotely and an alarm message can be sent to a remote location. Most of the wireless home automation systems are constrained by limited operating range. Such constraints can be overcome by using multi-hop communication system. This was the main motivation of the work presented in [33]. In this work a home control system (HCS) based on multi-hop mesh network is presented. Three different interfaces based on ZigBee, Bluetooth, and GPRS have been used in HCS. The hardware architecture and software protocol have also been proposed. Some techniques to save battery life and hence maximize operating life of such network have also been proposed in the same work. A ZigBee based embedded remote control system has been implemented in [35]. The main advantage of this system is that it provides the wireless communication capabilities on an embedded board rather than on a PC. This kind of embedded board has made system smaller in size and power efficient. The interference between the home appliances and the home network has been investigated in [36]. The authors have shown that the presence of the home appliances in a Personal Area Networks (PAN) environment significantly affect the performance of the home network system which also operates in the same frequency band. A ZigBee based home automation system and Wi-Fi network have been integrated in [37]. The home automation system has been implemented by using Texas Instrument's MCU device LM359B96 in [38]. Users can access the system by a dynamic webpage of LwIP TCP/IP protocol stack or GSM sms. The performances of a ZigBee based home automation system have been compared with those of other technology based (i.e., WiFi and Bluetooth) systems in [39]. The authors claimed that ZigBee based system has longer life compared to WiFi and Bluetooth based home automation system. An energy efficient wireless sensor network has been proposed for smart home in [40]. This system uses energy efficient sensors. This system is situation based self-adjusting to reduce the energy consumption in home automation system. An automatic embedded software generation framework has been proposed in [41] to create and evolve ZigBee applications. The main target is to enhance the quality of digital home living environments. This framework allows rapid deployment of the supporting software for energy control and sensing devices to monitor energy usage in a home at any time. The performances of ZigBee technology have been investigated in [42]. The performances of this system such as latency, received signal strength indicator, and round trip delay have been investigated in this work.

Voice control system for ZigBee based home automation has been introduced in [34]. Speaker independent automatic speech recognition technique has been used. A number of modes have been used for convenience namely button trigger mode, voice password trigger mode, and circle recognition mode. A user can use any of the modes depending upon the conditions. A low power voice control system for home automation system has been proposed in [43]. In this system ZigBee network receives voice command as input to an ARM9 controller, which converts the data into a required format to be used in the microcontroller. Finally, the system generates some control characters to switch ON/OFF the home appliances. A client server based voice control system for home automation has been presented in [44]. Voice command is captured by a client 
system and is sent to a server via Wi-Fi wireless network. The server system converts the voice command into a form that is used to control the home appliances. Microsoft Speech (SAPI) has been used in this work for implementing voice recognition system. Mobile based voice command control and monitoring system has been implemented in [45], in which artificial intelligence has been used for voice recognition system. A multi-layer feed forward neural network has been used. Another similar work has been presented in [46]. Two controlling methods have been proposed namely time and speech. For speech recognition Microsoft voice engine tool has been used. A wireless home automation system has been designed for physically challenged to control the home appliances in [47]. Fault identification system has also been integrated in this system to monitor the conditions of the appliances. To provide security RFID based authentication system has been used. The results presented therein show that the system can control up to 20 appliances and 40 voice commands can be recognized by this system. In another recent work [48] the authors implemented a ZigBee based home automation system that could recognize up to 1225 voice commands with a success rate of $80 \%$.

In this work we also presented voice controlled based wireless home automation system for elderly and people with disability. Our work is different from other related works in the following ways. We used National Instruments' LabView software. We have the following advantages due to LabView. The user interface is easier to design and implement. The system can be remotely controlled by a mobile or a computer. The system can easily be extended to include more appliances. The system is easy-to- install and configurable. Unlike other related system no expertise skill is required to install and configure the system. Microsoft's rich voice recognition library has been included in our work. The system needs to be trained only one time. Once it is trained, the system can system can recognize the voice commands independent of vocabulary size, noise, speaker characteristics or accent. The data acquisition is performed by using DAQ provided by the National Instruments. The use of DAQ simplifies the hardware implementations of this proposed system compared to other existing similar systems.

\section{SYSTEM MODEL}

The system model of this work is shown in Figure1. The system model consists of the following basic components (i) automatic speech recognition system, (ii) control units, (iii) wireless system, and (iv) application and home appliances.

Automatic speech recognition (ASR) system can be defined as an independent and computer-driven transcription of spoken language that allows a computer to identify the spoken words captured from a microphone or telephone and convert it into written texts. The main components of an ASR are (i) a microphone, (ii) speech recognition software, (iii) a computer, and (iv) a sound card. The ultimate goal of ASR is to allow a computer to recognize in real-time, with $100 \%$ accuracy, all words that are spoken by any person, independent of vocabulary size, noise, speaker characteristics or accent. Through a speech recognition program/application, the computer is able to process words one says and turn them into text that is displayed on the screen. There have been many research activities on the speech recognition system. The fundamental reasons of these research activities are (i) accessibility for the deaf and hard of hearing, (ii) cost reduction through automation, and (iii) searchable text capability. 


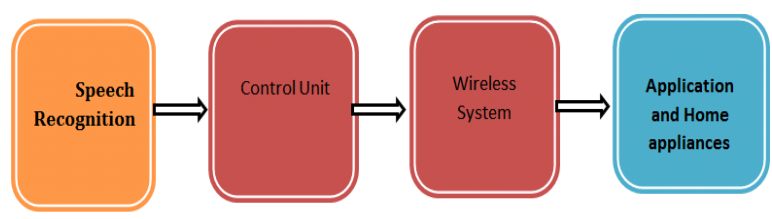

Figure 1. The system model

The control unit (CU) coordinates the components of a computer system. It fetches the code of all of the instructions in a program. It directs the operation of the other units by providing timing and control signals. All computer resources are managed by the CU. It directs the flow of data between the Central Processing Unit (CPU) and the other devices. There are different types of control program available including MatLab, $\mathrm{C}++$, and LabView. In this work we choose LabView because of the following reasons: (a) it is easier to build a large program piecewise using small amounts of code, (b) it is easy to control the interfaces between hardware, (c) it is easy to find compatible hardware in the market, (d) it is simple to integrate with other hardware and software, and (d) it is easy to create the user-interface.

Wireless networks and sensors are playing important roles in emerging pervasive computing technologies that are required for the realization of smart homes. Effectively all wireless technologies that can support some form of remote data transfer, sensing, and control are candidates for inclusion in the smart home portfolio. In this work we used ZigBee wireless system. Compared to other wireless systems like Bluetooth and Wi-Fi, ZigBee has some advantages as follows: (a) ZigBee aims at automation whereas other two technologies aim at the connectivity of mobile devices in close proximity, (b) ZigBee uses low data rates, and consumes low power, (c) ZigBee networks support devices with longer range, and (d) ZigBee network is a robust network and that is easily scalable.

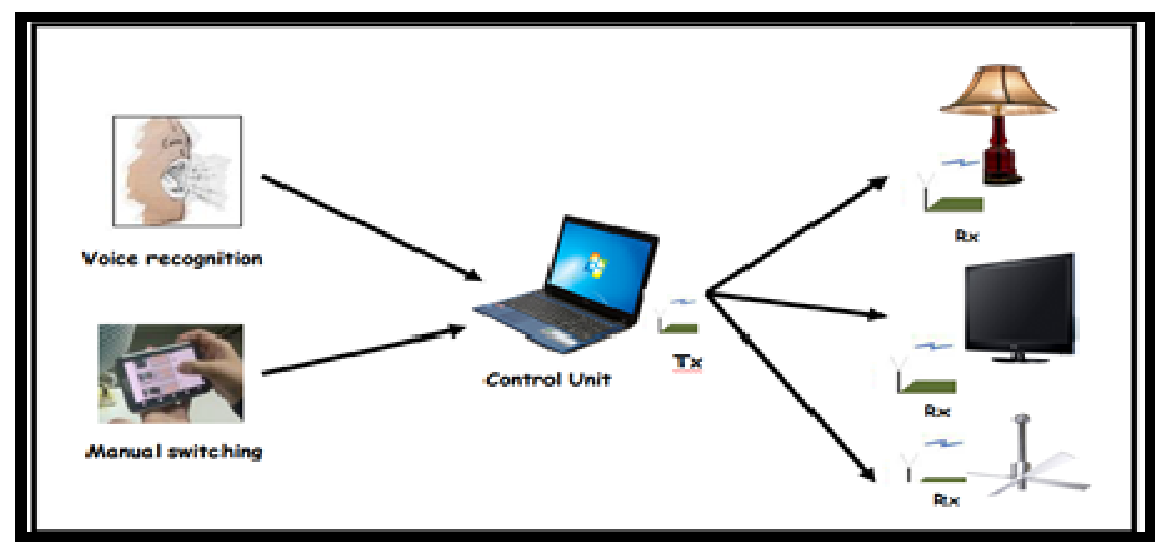

Figure 2. System operation

Applications and home appliances are the last step of the proposed system. Each device connected with the network must have a switching circuit to turn it On\ OFF. The status of each device is monitored by using toggle circuit. In addition to the switching devices a Data Acquisition Card (DAQ) has been used in this work. A typical commercial DAQ card contains $\mathrm{ADC}$ and DAC that allow input and output of analog and digital signals in addition to digital 
input/output channels. There are many types of DAQ cards are available to provide interface between the LabView software and the hardware. In this project NI USB 6008 DAQ card has been used.

\section{SYSTEM OPERATION}

The basic system operation of the proposed system is shown in Figure 2. A user has two options for controlling the home appliances (a) by using manual switching and (b) by using voice recognition. The control unit is an interface program that must satisfy the following two conditions (a) the output from the interface program is forwarded to a wireless transmitter and sent to a receiver through wireless channel, and (b) the receiver at the appliances accept the receive signal to turn ON or OFF the device. The front panel of the LabView program is shown in Figure 3. The system operation can be described based on this front panel in the following steps:

- The voice command is captured by using a microphone and sent to the computer.

- Voice recognition is performed by using LabView with the help of Microsoft's rich speech library.

- Upon recognition of the voice command control characters are generated and sent through ZigBee wireless communication protocol to specified application address.

- At receiver side, application can turn ON or OFF a relay controlling circuit depending on the received controlled characters.

- A hardware switching toggle circuit is added to simplify the switching process and to allow for ON/ OFF switching with a single word voice command.

- As an additional feature the system can be controlled using smart devices.

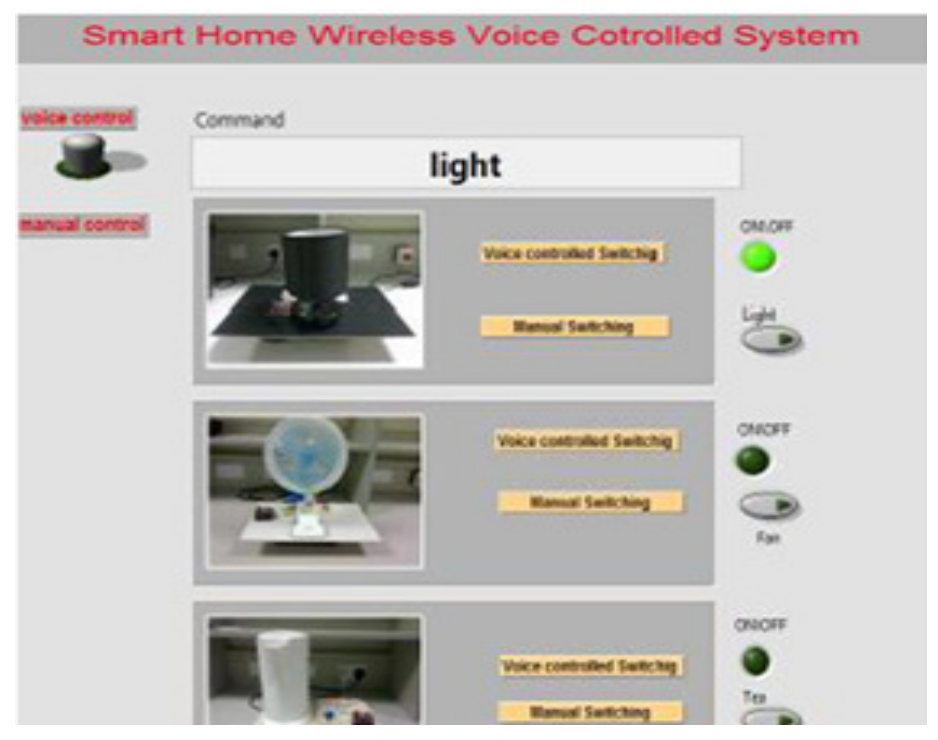

Figure 3. The front panel of the program.

The circuit block diagram of the transmitter system is shown in Figure 4. The speech recognition is dealt with LabVIEW. So the program recognizes the speech with library inside it. Then the output from LabView is sent to the DAQ. The DAQ connects the microcontroller and transmitter. 
The transmitter sends commands to the wireless receiver that is connected with the switching circuit.

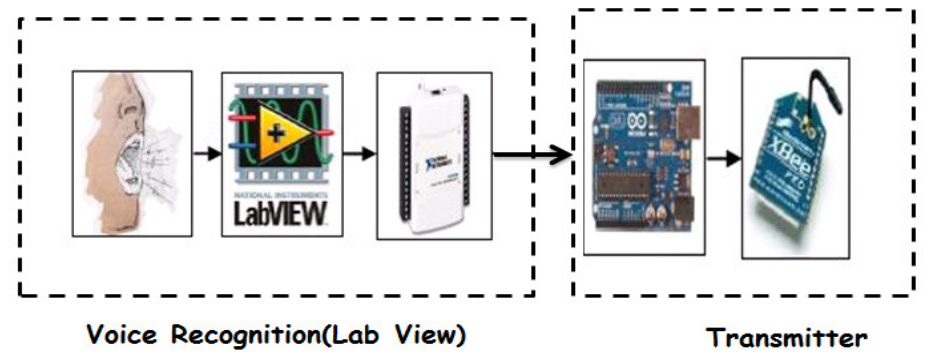

Figure 4. Transmitter Circuit.

The LabView takes input commands from the voice recognition system, compares the command with each stored command inside the library of LabView. The comparison is done in terms of the frequency. If the frequencies of the commands are matched, the LabView will generate an output pulse that is sent via DAQ. The detail operation of the transmitter is illustrated in Figure 5.

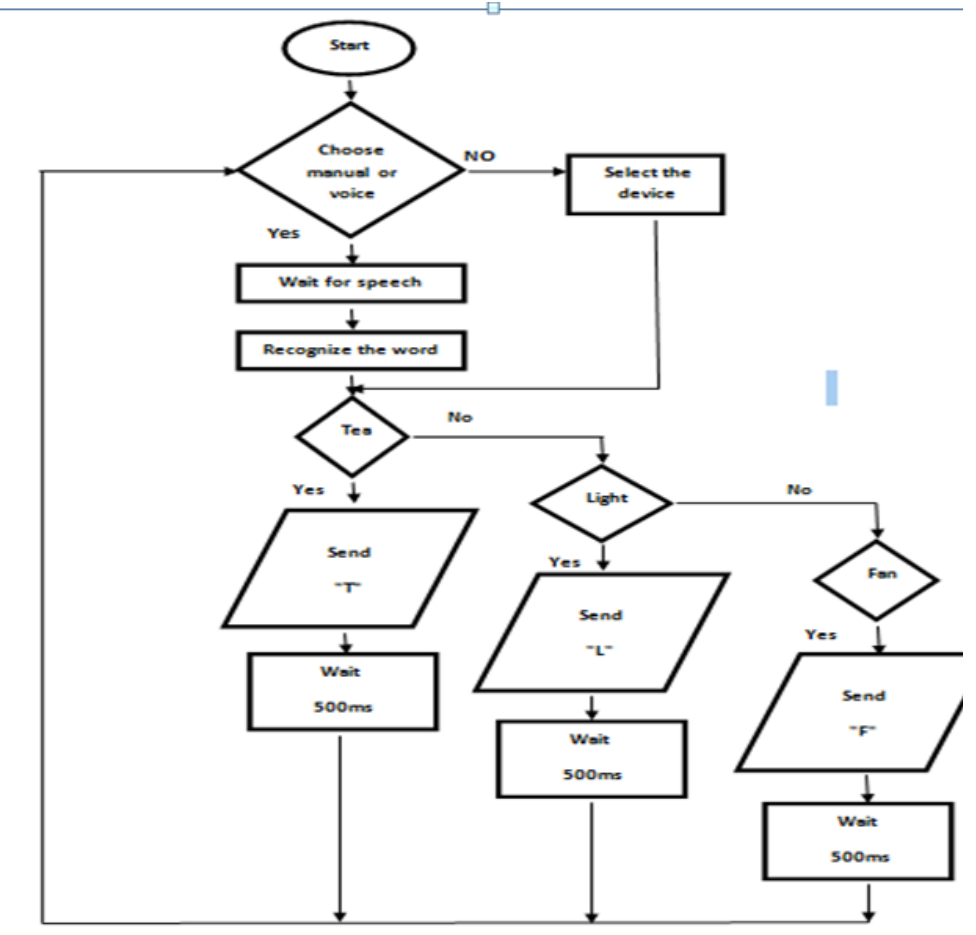

Figure 5 The flowchart for the operation of the transmitter. 


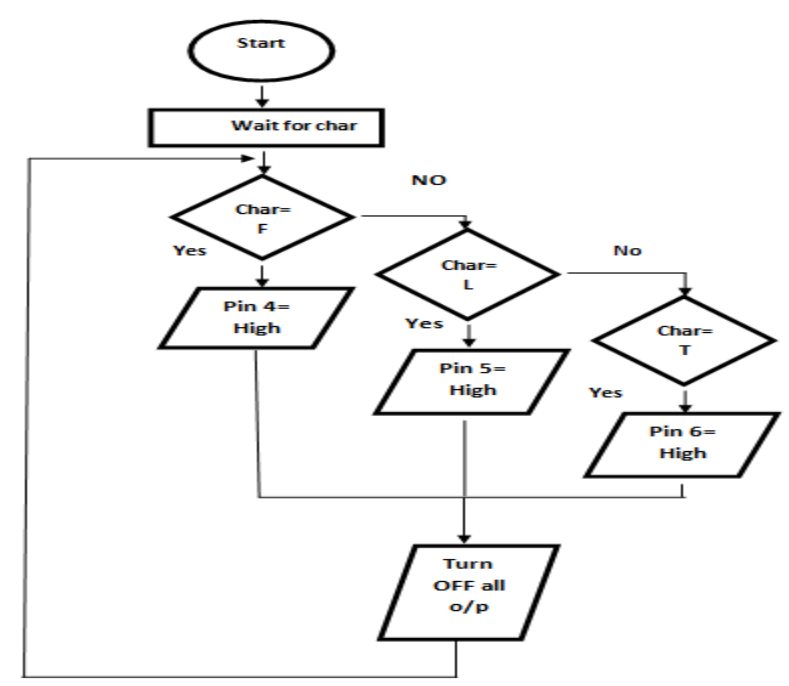

Figure 6 The flowchart for the operation of the receiver

The wireless transmission unit consists of Xbee and Arduino. The Arduino is programmed in such way that if it receives the pulse code 100 from labVIEW program, it will generate a character "T". If it receives the pulse code 010 , it will generate a character "L". If it receives the pulse code 001, it will generate a character " $F$ ". Then the program will wait for $500 \mathrm{~ms}$ after transmitting a new data. The receiver module is connected with the switching circuit of the appliances. We connect the pins of the receiver module to the home appliances as follows: Pin 4 to the fan, Pin 5 to the light, and Pin 6 to the tea machine. The program waits for receiving a character. For example, if the program receives character " $F "$ it will turn $O N$ the fan. If the program again receives the same character, it will turn OFF the fun by using the switching circuit. Similar procedure is followed for switching ON or OFF the other appliances (i.e., light and tea machine). Figure 6 shows the flowchart of the processes of wireless receiver.

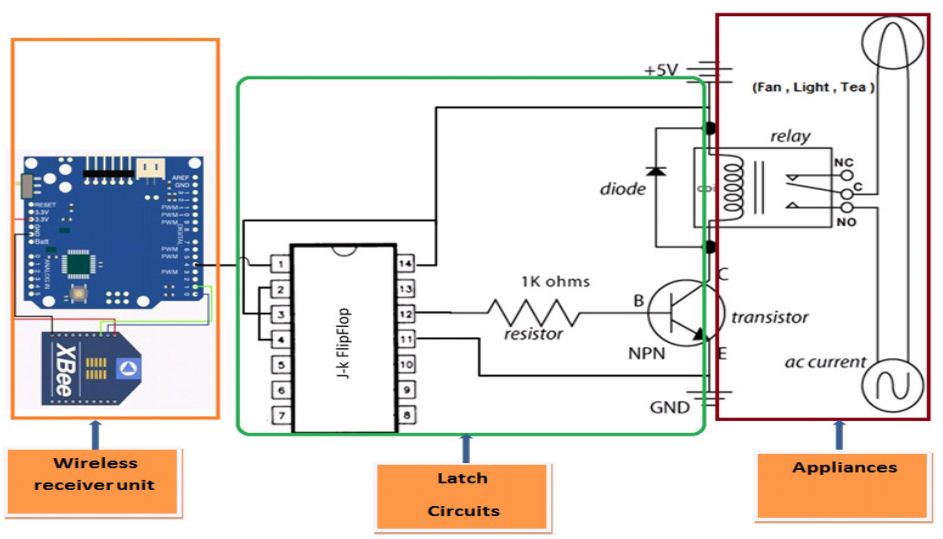

Figure 7. The latch circuit 
International Journal of Wireless \& Mobile Networks (IJWMN) Vol. 6, No. 1, February 2014

In this work, we use the same word to turn ON/OFF the appliances. Since the LabView cannot turn ON/OFF the appliances by using the same word, we use a switching circuit to switch an appliance between the two states. It means that if a character is received for the first time, the program will turn on the device. If the same character is received for the second time, the program will turn off the device. We have designed a latch circuit for this type of switching operation. The latch circuit is connected with the relay that switches ON\OFF the device. The latch circuit is shown in Figure 7. The latch is a basic storage element in a sequential logic. Flipflops and latches are the fundamental building blocks of digital electronics systems used in computers, communications, and many other types of systems. The latch circuit used in this work has been implemented by using a J-K flip-flop. In electronics, a flip-flop or latch is a circuit that has two stable states and can be used to store state information. The circuit can be made to change the state by applied signals. When used in a finite-state machine, the output and next state depend not only on its current input, but also on its current state (and hence previous inputs

\section{CONCluSions}

In this work, we have implemented a voice controlled Zigbee-based home automation system. We used speech recognition system to implement this work. The LabView software has been used to implement the voice recognition system. The main advantage of the system is that it does require training of voices for only one time. At the same time LabView software has been used to support human-computer interactions to realize multiple functions. The system is designed for elderly and disabled people so that they can monitor and control the home appliances with their limited ability. The wireless part of the system has been implemented by using Zigbee RF modules. Hence, the system is highly efficient and it consumes low power. This system can be easily extended to remotely control the home appliances through smart devices like iPhone/ iPod and others phones so that one can remotely check the status of the home appliances and turn ON or OFF the same. The proposed system has been tested with three home appliances. But, it can be easily expanded to include more home appliances.

\section{REFERENCES}

[1] A.J. Bernheim Brush, Bongshin Lee, Ratul Mahajan, Sharad Agrawal, Stefan Saroiu, Collin Dixon (2011), "Home Automation in the Wild: Challenges and Opportunities", Proceedings of ACM CHI Conference on Human Factors of Computing System, May 7-12, Vancouver, BC, Canada

[2] Richard Harper (2003) "Inside the Smart Home", Springer-Verlag London Limited 2003

[3] Juile A Kietz, Shwetak N. Patel, B rian Jones, Ed Price, Elizabeth D. Mynatt, Greogory A. Abowd (2008) "The Georgia Tech aware Home", The 26th CHI Conference, April 5-10, Florence, Italy ,2008, pp. 3675-3680

[4] Tiiu Koskela and Kaosa-vaananen-vainio-Mattila (2004) " Evolution towards smart home environments:empirical evaluation of three users interfaces, International Journal of Personal and Ubiquitous Computing, July, Vol. 8, No. 3-4, pp. 234-240

[5] Daine J. Cook and Sajal K. Das (2005) "Smart Home Environments: Technology, Protocols, and applications", Wiley series of Parallel and Distributed Computing (kindle Edition), pp. 273-294

[6] Tom Rodden and Steve Benford (2003) "The evolution of buildings and implications for the design of ubiquitous domestic environments", Proceedings of the SIGCHI Conference on Human Factors in Computing Systems", April 5-10, Fort Lauderdale, Florida, pp. 9-16

[7] Home security systems, home security products, and home alarm systems available at http://www.adt.com

[8] ABI Research on home automation available at http://www.abirearch.com

[9] Vijay Saraswat, Bard Bloom, Igor Peshansky, Olivier Tardieu, and David Grove, “ X10 Language Specifications Version 2.4”, September (2013) available at http://x10.sourceforge.net/d ocumentation /languagespec/x10-240.pdf 
International Journal of Wireless \& Mobile Networks (IJWMN) Vol. 6, No. 1, February 2014

[10] Insteon White Paper: The Details available at http://www.insteon.com/

[11] Y. Kyselytsya and Th. Weinzierl, " Implementation of the KNX Standard" available at http://www.weinzierl.de/

[12] The Z-Wave technology available at http://www.Z-wavealliance.org/technology

[13] ZigBee Home Automation available at http://www.zigbee.org/Standards/

[14] IoBridge Connect available at http://connect.iobridge.com/docs/

[15] Janko Roettgers, “Time for Google's Android @ Home to Make a New Splash" available at http://www.businessweek.com/articles/2013-05-08/

[16] W. Keith Edwards, Rebecaa E. Grinter, Ratul Mahajan, and David Wetherall (2011) “ Advancing the state of home networking", Communication of ACM, Vo. 5, No.6, July, pp. 62-71

[17] Forest and Sullivan, "North American Home Automation Market", available at http://www.forest.com/sublib

[18] Intile S.S. (2002) "Designing a home of the future", IEEE Pervasive Computing, Vol. 1, No.2, pp. 7682

[19] Scott Davidoff, Min Kyung Lee, Charles Yiu, John Zimmerman, and Anind K. Dey (2006) "Principles of smart home control", Proceedings of the 8th International Conference on Ubiquitous Computing, 17-21 September, pp. 19-34.

[20] Frost and Sullivan, "Innovation and Affordability open up home automation to alarge audience" available at http://www.frost.com/prod/servlet/press-release- pag?docid=273090581

[21] Carles Gomez and Joseph Paradells (2010) "Wireless Home Automation Networks: A survey of Architectural and Technologies”, IEEE Communication Magzine, June (2010), pp. 92-101

[22] http://www.radiocomms.com.au/products/42985-Wavenis-Open-Standard-Alliance

[23] C. Perkins, E. Belding-Royer, and S. Das, "Ad hoc On-Demand Distance Vector (AODV) Routing" available at http://www.ietf.org/rfc/rfc3561.txt

[24] ZigBee Alliance, “ ZigBee Home Automation Public Application Profile”, version 25, vol. 1.0, October 2007.

[25] Dechuan Chen and Meifang Wabg (2006) " A home security ZigBee network for remote monitoring application", Proceedings of IET International Conference on Wireless, Mobile, and Multimedia networks", November 6-9, Hangzhou, China, pp. 1-4

[26] Woo-Choo Park and Myung-Hyun Yoon (2006) "The Implementation of Indoor Location System to Control ZigBee Home Network", Proceedings of International Joint Conference SICR-ICASE" October 18-21, Busan, South Korea, pp. 2158-2161

[27] Kawamoto, R., Emon , T., Sakata, S., and Youasa, K. (2007) "Energy efficient sensor control scheme for Home Networks based on DLNA-ZigBee Gateway Architecture”, Proceedings of the First International Global Information Infrastructure Symposium, July 2-6, Marrakech, Morocco, pp. 7379

[28] Zhang Shunyang, Xu Du, Jiang Yongping, Wang Riming, " Realization of Home Remote Control networks based on ZigBee (2007) "Proceedings of the 8th International Conference o Electronic Measurements and Instrumentations, August 16-18, Xiang, China, pp. 4-344-4-348

[29] Jui-Yu Chang, Tao-Yuan, Min-Hsing Hung, and Yen-Wei Chang (2007) " A ZigBee based Power Monitoring System with Direct Load Control Capabilities”, Proceedings of IEEE International Conference on Networking, Sensing, and Control, April 15-17, London, pp. 895-900

[30] Ying-Wen Bai and Chi-Huang Hang (2008) " Remote Power ON/OFF control and current measurement for home electric outlets based on a low-power embedded board and ZigBee Communication", Proceedings of IEEE International Symposium on Consumer Electronics, April 1416, Vilamoura, pp.1-4

[31] Jan Hou, Wu Cang dong, Zhongjia Yuan, Jiyuan Tan (2011), “ Research of Intelligent Home Security Surveillance System based on ZigBee", Proceedings of the Initial Symposium on Intelligent Information Technology Application Workshops, December 21-22, Shanghai, pp. 554-557

[32] Jianfeng Wu, and Hubin Qin (2008) “ The deisgn of wireless intelligent home system based on ZigBee", Proceedings of the 11th IEEE International Conference on Communication Technology, November 11-12, HagZhau, pp. 73-76

[33] Fei Ding, Guangming Song, Jianing Li, and Higuo Song (2008) "A ZigBee Based Mesh Network for Home Control", Proceedings of International Workshop on Geoscience and Remote Sensing", Shanghai, 2008, Vol. 1, pp. 744-740 
International Journal of Wireless \& Mobile Networks (IJWMN) Vol. 6, No. 1, February 2014

[34] Jieming Zhu, Xuecai, Yucang Yang, and Hang Li (2010) “ Developing a voice control system for ZigBee based home automation”, Proceedings of IEEE International Conference on network Infrastructure and Digital Content, September 24-26, Beijing, 2010, pp. 7737-741

[35] Cui Chenguyi, Zhao Guannan, and Jin Mingle (2010) “A ZigBee based embedded remote control System, Proceedings of the 2nd International Conference on Signal Processing Systems, 5-7 July, 2010, Dalian, pp. v3-373-376

[36] Simek, M., Fuchs, M, Mraz, L., and Morvek, P. (2011) “ Measurement of LowPAN Network coexistence with Home Microwave Appliances in Laboratory and Home Environments", Proceedings of International Conference on Broadband and Wireless Computing, October 26-28, 2011, Bercelona, pp. 292-299

[37] Ming Zhi Wu, Wei-Tsang Lee, Ren-JiLino, Chaye, G. (2012) “ Development and validation of an integrated dynamic security monitoring platform," Proceedings of the 6th International Conference on Genetic and Evolutionary Computing, August 25-28, 2012, pp. 524-517, Kotakushu, 2012

[38] Chunglong Zhang, Min Zhang, Young Sheng Su, and Weillian Wang (2012), " Smart home design based on ZigBee wireless sensor network", Proceedings of the 7th International ICST Conference on Communications and networking in China, Kun Ming, pp. 463-466

[39] Rathod K., Parikh, N., and Shah, V. (2012) “ Wireless automation using ZigBee protocols”, Proceedings of the 9th International Conference on Wireless and Optical Communication, Indere, September 20-22, 2012, pp.1-5

[40] Jin Sung Byum, Boungju Jeon, Junyoung Noh, and Youngil Kim (2012) “ An Intelligent selfadjusting sensor for smart home services based on ZigBee Communiation", IEEE Transaction on Consumer Eletronics, Vol. 58, No. 3, pp. 799-802

[41] Chihhsiong Shih and Bwo-cheng Liang (2012) "A model driven software framework for ZigBee based energy saving systems", Proceedings of the 3rd International Conference on Intelligent Systems, modeling and Simulation, February 8-10, Kota Kinabaiu, pp. 487-492

[42] Karia Deepak, Jaypal Bavisker, Raj Makwana and Panchat Niraj (2013) "Performance analysis of ZigBee based load control and power monitoring system", Proceedings of the International Conference on Advances in Computing, Communications, and Informatics, Myshore, August 22-25, 2013, pp. 1779-1484.

[43] Y.B. Krishna and S. Nagendram (2012) "ZigBee Based Voice Control System for Smart Home”, International Journal on Computer Technology and Applications, Vol. 3, no. 1 (2012), pp. 163-168

[44] B. Mardiana, H. Hazura, S. Fauziyah, M. Zahariah, A.R. Hanim, and M. K. Noor Shahida (2009) "Home Appliances control Using Speech Recognition in Wireless Network Environment", Proceedings of the International Conference on Computer Technology and Development, pp. 285288.

[45] N.P. Jawarkar, V. Ahmed, and R.D. Thakare (2007) “ Remote Control using mobile through spoken commands", Proceedings of IEEE International Consortium of Stem Cell Networks”, 2007, pp. 622 625.

[46] S.M.A. Haque, S.M. Kamruzzaman, and Md. Islam (2006) “ A system for Smart Home Control of Appliances based on timer and Speech Interaction", Proceedings of the 4th International Conference on Electrical Engineering, January 26-28, pp. 128-13

[47] Gananasekar, A.K., Jayarelu, P., and V. Nagrajan (2012) “ Speech recognition based wireless automation of home with fault identification for physically challenged" , Proceedings of International Conference on Communications and Signal Processing, April 4-5 2012, Chennai, pp. 128-132

[48] Al Shueili, H. and Sen Gupta G, Mukhopadhyaya (2001) "Voice recognition based wireless home automation system", Proceedings of the International Conference on Mechatronics , May 17-19, Kualalumpur, pp. 1-6

[49] http://whqlibdoc.who.int/hq/2011/WHO_NMH_VIP_11.01_eng.pdf

[50] http://www.un.org/esa/population/publications/worldageing19502050/ 
International Journal of Wireless \& Mobile Networks (IJWMN) Vol. 6, No. 1, February 2014

\section{AUTHORS}

Thoraya Obaid Saeed is with the electrical engineering department of Ajman University of Science and Technology, United Arab Emirates (UAE). She started her undergraduate study in Electrical Engineering with major in Communication in 2009. She completed her Bachelor of Science in Engineering in 2013. Her research interests are in sensor network, ZigBee and Home Automation. She is also interested in reading and photography.

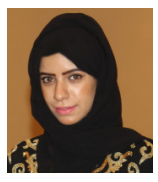

Haliemah Rashed is with the electrical engineering department of Ajman University of Science and Technology, United Arab Emirates (UAE). She started her undergraduate study in Electrical Engineering in 2009. She completed her Bachelor of Science in Engineering with major in communication in 2013. Her research interests are in wireless communication, sensor network, ZigBee and Home Automations.

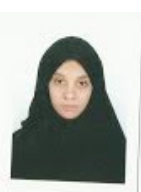

Ali Abou-Elnour received the $\mathrm{PhD}$ degree in Electrical Engineering from Technical University Hamburg Harburg (THUU) Germany. He is a Professor at the Electrical Engineering Department, College of Engineering, Ajman University of Science and Technology (AUST) United Arab Emirates since 2008. Since 2010 he is the Deputy Dean of the college of Engineering, of AUST in Fujairah. He is now the Director of AUST-Fujairah, UAE. His current research interests include modeling of semiconductor devices, ultra-small,

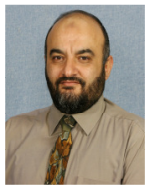
nano-electronic and microwave semiconductor devices, magnetic sensors, energy management systems, wireless monitoring and controlling systems, healthcare monitoring systems, smart home systems, and solar and renewable energy systems, where he has participates and coordinated research projects and authored and co-authored papers in journals and conferences

Muhammad Rehan Siddiqi completed his Bachelor of Science in Electrical Engineering/Electronics from Ajman University of Science and Technology, United Arab Emirates. Currently he is with the electrical engineering department of the same university as a teaching assistant. He is also pursuing his M.S in Engineering Management at University of Wollongong, Dubai. His research interests are in electronic circuit design, wireless communication, smart home system.

Musaab Hasan received the B.Sc. in electrical engineering/ communication from Ajman University of Science \& Technology, United Arab Emirates with an AGPA of 4/4, UAE, in 2012. .After graduation, he stayed in the same university as teaching assistant. He carried out research in networking and wireless communication and participated in several competitions

Mohammed Tarique completed his PhD from Lamar University, Texas,USA. Currently he is with the electrical engineering department of Ajman University of Science and Technology, United Arab Emirates. He has six years of industrial experience and seven years of teaching experience. His research interests are in wireless communication, digital signal processing, sensor networks, ad hoc networks.
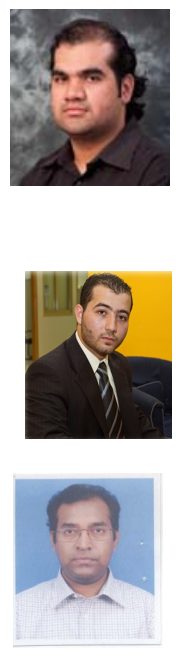